\title{
Magnetic Field Mapping using Off-Axis Electron Holography in the Transmission Electron Microscope
}

\author{
Fengshan Zheng ${ }^{1}$, András Kovács ${ }^{1}$, Thibaud Denneulin ${ }^{1}$, Jan Caron ${ }^{1}$, Teresa Weßels ${ }^{1}$, Rafal E. Dunin-Borkowski ${ }^{1}$ \\ ${ }^{1}$ Ernst Ruska-Centre for Microscopy and Spectroscopy with Electrons and Peter Grünberg Institute, Forschungszentrum Jülich
}

\section{Corresponding Authors}

Fengshan Zheng

f.zheng@fz-juelich.de

András Kovács

a.kovacs@fz-juelich.de

\section{Citation}

Zheng, F., Kovács, A., Denneulin, T.,

Caron, J., Weßels, T., Dunin-

Borkowski, R.E. Magnetic Field

Mapping using Off-Axis Electron

Holography in the Transmission Electron

Microscope. J. Vis. Exp. (166), e61907,

doi:10.3791/61907 (2020).

\section{Date Published}

December 4, 2020

\section{DOI}

$10.3791 / 61907$

URL

jove.com/video/61907

\section{Abstract}

Off-axis electron holography is a powerful technique that involves the formation of an interference pattern in a transmission electron microscope (TEM) by overlapping two parts of an electron wave, one of which has passed through a region of interest on a specimen and the other is a reference wave. The resulting off-axis electron hologram can be analyzed digitally to recover the phase difference between the two parts of the electron wave, which can then be interpreted to provide quantitative information about local variations in electrostatic potential and magnetic induction within and around the specimen. Off-axis electron holograms can be recorded while a specimen is subjected to external stimuli such as elevated or reduced temperature, voltage, or light. The protocol that is presented here describes the practical steps that are required to record, analyze, and interpret off-axis electron holograms, with a primary focus on the measurement of magnetic fields within and around nanoscale materials and devices. Presented here are the steps involved in the recording, analysis, and processing of off-axis electron holograms, as well as the reconstruction and interpretation of phase images and visualization of the results. Also discussed are the need for optimization of the specimen geometry, the electron optical configuration of the microscope, and the electron hologram acquisition parameters, as well as the need for the use of information from multiple holograms to extract the desired magnetic contributions from the recorded signal. The steps are illustrated through a study of specimens of B20type $\mathrm{FeGe}$, which contain magnetic skyrmions and were prepared with focused ion beams (FIBs). Prospects for the future development of the technique are discussed.

\section{Introduction}

Magnetic nanostructures are increasingly used in applications that include nanoscale logic, storage, and spintronic devices $^{1,2,3,4,5}$. A local understanding of the magnetic properties of the constituent materials requires the development of techniques for magnetic characterization with nanometer $(\mathrm{nm})$ spatial resolution, both in projection 
and in three dimensions, ideally while the specimen is subjected to external stimuli such as elevated or reduced temperature, applied voltage, or light. Currently-available magnetic characterization techniques include magnetooptical Kerr effect microscopy, magnetic force microscopy, spin-polarized scanning tunneling microscopy, spin-polarized low-energy electron microscopy, X-ray magnetic circular dichroism, X-ray holography, and scanning transmission Xray microscopy $6,7,8,9,10,11$.

In transmission electron microscopy, magnetic characterization techniques include the Fresnel and Foucault modes of Lorentz microscopy, off-axis electron holography, differential phase contrast (DPC) imaging, and electron magnetic circular dichroism (EMCD) $6,7,12,13,14$. The focus of this paper is on the technique of off-axis electron holography, which is capable of providing real-space quantitative measurements of magnetic fields inside and around nanoscale materials with sub-5-nm spatial resolution, both in projection and, when combined with electron tomography, in three dimensions ${ }^{13,14}$.

In the TEM, a highly accelerated electron beam is passed through an electron-transparent (usually solid) specimen to provide access to its crystallographic, chemical, electronic, and/or magnetic structure with a spatial resolution that can reach the atomic scale. Typically, a thin $(<100 \mathrm{~nm})$ specimen is irradiated with electrons that are emitted from an electron gun and accelerated by $60-300 \mathrm{kV}$ in a highvacuum $\left(<10^{-5} \mathrm{~Pa}\right)$ column. Electromagnetic lenses are used to focus electrons onto the specimen and subsequently onto one or more detectors. The electrons interact strongly with the atomic potentials in the specimen and with electromagnetic fields within and around it. Although this information is encoded in the electron wavefunction, an in-focus bright- field or dark-field TEM image records only variations in the intensity of electrons that reach a detector, while information about their phase shift is lost. This so-called "phase problem" is also encountered in X-ray and neutron experiments.

One of the techniques that allows the measurement of the phase shift of the electron wavefunction is off-axis electron holography. Further details about fundamental aspects of electron wave functions are available elsewhere ${ }^{15}$. The concept of electron holography was first proposed by Dennis Gabor in 1948 to overcome limitations in the spatial resolution of electron microscopy due to aberrations of the primary imaging lens of the microscope ${ }^{16}$. The technique allows information about both the amplitude and the phase of an electron wave to be recorded. It has been readily available for commercial electron microscopes since the 1990s, in part owing to developments in field emission gun technology. Although more than 20 variations of electron holography have been described, the most popular and versatile type is currently the TEM mode of off-axis electron holography ${ }^{17}$ for electromagnetic field mapping with high spatial resolution $18,19,20,21,22,23$.

The TEM mode of off-axis electron holography involves the formation of an interference pattern or hologram by overlapping two parts of an electron wave (Figure 1A), one of which has passed through a region of interest on the specimen and the other is a reference wave ${ }^{24}$. The phase shift $\Phi$ can be retrieved digitally from a recorded off-axis electron hologram and interpreted to provide quantitative information about local variations in the electrostatic potential and magnetic vector potential by using Equation $\mathbf{1}^{25}$,

$$
\begin{aligned}
& \phi(x, y)= \\
& C_{E} \int_{-\infty}^{+\infty} V(x, y, z) d z-\frac{2 \pi e}{h} \int_{-\infty}^{+\infty} A_{z}(x, y, z) d z,
\end{aligned}
$$


where $C_{E}$ is an interaction parameter that depends on the microscope accelerating voltage $\left(C_{E}=6.53 \times 10^{6} \mathrm{rad} /(\mathrm{Vm})\right.$ at $300 \mathrm{kV}), V(x, y, z)$ is the electrostatic potential, $A_{z}(x, y, z)$ is the $z$ component of the magnetic vector potential, $z$ is parallel to the incident electron beam direction, $e$ is an elementary unit of charge, and $h$ is Planck's constant. The electrostatic and magnetic contributions to the phase shift can be separated, for example, by combining information from electron holograms recorded before and after turning the specimen over, from electron holograms recorded below and above the magnetic Curie temperature of the specimen, or from electron holograms recorded at different microscope accelerating voltages ${ }^{13,26}$. Once the magnetic contribution to the phase shift $\Phi_{m}$ (i.e., the second term on the right side of Equation 1) has been retrieved, the in-plane magnetic induction projected in the electron beam direction, $\boldsymbol{B}_{p}$, can be obtained from its first derivatives by using Equation 2,

$B_{p}=\left(B_{p, x}, B_{p, y}\right)=\frac{h}{2 \pi e}\left(-\frac{d \phi_{m}}{d y}, \frac{d \phi_{m}}{d x}\right)$

where $B_{p, x}=\int_{-\infty}^{+\infty} B_{x}(x, y, z) d z$ and

$B_{p, y}=\int_{-\infty}^{+\infty} B_{y}(x, y, z) d z$

A magnetic induction map can then be displayed using contours and colors to provide a visual representation of the magnetic field of a thin film or nanostructure $26,27,28,29,30,31$, as described below. Magnetic phase images and magnetic induction maps should always be interpreted with great care: first, because they represent two-dimensional projections of three-dimensional (3D) magnetic vector fields; second, because they are insensitive to out-of-plane components of the magnetic field $B_{Z}$; and third, because they combine information from magnetic fields that are present both inside and outside the specimen. Fortunately, it is now possible to recover $3 \mathrm{D}$ magnetic information from tomographic tilt series of magnetic phase images by using either backprojection-based $32,33,34,35,36,37$ or model-based $38,39,40$ reconstruction algorithms.

Transmission electron microscopic studies of the magnetic properties of materials are usually carried out with the specimen in magnetic-field-free conditions, i.e., after turning off the conventional microscope objective lens and using either a non-immersion Lorentz lens or the transfer lenses of an image aberration corrector as the primary imaging lens. The use of an additional specimen stage located between the condenser and objective lenses ${ }^{41}$ or a double objective lens system to cancel the magnetic field at the specimen position $^{42}$ can also help achieve magnetic-fieldfree conditions. The recording of images with the specimen located in magnetic-field-free conditions is often referred to as Lorentz microscopy. Lorentz transmission electron microscopy is a rapid technique to check the magnetic state of the specimen in the presence of external stimuli. However, it is usually only applied qualitatively and is not readily applicable to studies of magnetic fields in the smallest nanostructures, in part due to the presence of Fresnel fringes from local changes in specimen thickness. Depending on the specification of the microscope and the specimen of interest, a variety of different imaging, diffraction, or spectroscopy techniques (e.g., DPC imaging and EMCD) can be used to perform magnetic characterization in transmission electron microscopy. 
Off-axis electron holography is often applied in combination with the simpler, albeit less quantitative, technique of Fresnel defocus imaging (i.e., the Fresnel mode of Lorentz microscopy), particularly for studies of magnetic domain walls. Just as for off-axis electron holography, the contrast in Fresnel defocus images arises from the refraction of electrons by the in-plane component of the magnetic field within and outside the specimen. To a first approximation, an in-plane magnetic field $B_{x y}$ in a specimen of thickness $t$ results in deflection of the incident electron beam by an angle $\vartheta=\frac{e \lambda}{h} B_{x y} t$, where $\lambda$ is the (relativistic) electron wavelength. When using Fresnel defocus imaging, the positions of magnetic domain walls are revealed as lines of dark or bright intensity in defocused bright-field images. Phase information can be recovered from such images by solving the transport-of-intensity equation ${ }^{43}$. However, a lack of knowledge of boundary conditions at the edges of the field of view can result in errors in the reconstructed phase.

In contrast, when using the Foucault mode of Lorentz microscopy, an aperture is used to allow only electrons that have been deflected in a specific direction to contribute to image formation. It should be noted that DPC imaging in scanning transmission electron microscopy and the Fresnel mode of Lorentz microscopy record signals that are approximately proportional to the first and second derivatives of the phase shift of the electron wave, respectively. As a result, they can contain strong contributions from local changes in specimen thickness and composition, which can dominate magnetic contributions to the contrast ${ }^{6,7}$.

From an experimental perspective, the TEM mode of offaxis electron holography requires the use of an electrostatic biprism, which usually takes the form of a thin conductive wire that is positioned close to one of the conjugated image planes in the microscope. The application of a voltage to the biprism to overlap the object and reference electron waves (Figure 1A) results in the formation of an electron hologram, which can be recorded on a charge-coupled device (CCD) camera or a direct electron counting detector ${ }^{44}$.

The condenser lens stigmator settings are typically adjusted to make the electron beam highly elliptical to maximize the lateral coherence of the beam in a direction perpendicular to the biprism, while retaining a sufficient number of electron counts. The region of interest on the specimen is positioned so that it covers part of the field of view, while a reference hologram is usually obtained from an adjacent region of vacuum or a region of thin clean support film. The experiments that are described below were carried out in an image-aberration-corrected TEM operated at 300 $\mathrm{kV}$. This microscope has a large $(11 \mathrm{~mm})$ pole-piece gap and is equipped with two electron biprisms (Figure 1B). In these experiments, only one of the biprisms was used to record electron holograms. The advantages of using multiple biprisms are described elsewhere ${ }^{45,46}$. Fresnel defocus images and off-axis electron holograms were recorded using either a conventional $2 \mathrm{k} \times 2 \mathrm{k}$ CCD camera or a $4 \mathrm{k} \times$ $4 \mathrm{k}$ direct electron counting detector. Lorentz mode was set up by adjusting the objective lens to a small negative excitation to achieve a magnetic-field-free environment at the specimen position by compensating for the residual magnetic field of the objective and nearby lenses. The first transfer lens of the image corrector unit was then used as a nonimmersion imaging lens. Specimens could be imaged either at remanence (in zero magnetic field) or in the presence of a pre-calibrated magnetic field ${ }^{47}$, which could be applied by exciting the conventional microscope objective lens. The twin structure of the objective lens in this microscope allows 
magnetic fields in the range from $-150 \mathrm{mT}$ to $1.5 \mathrm{~T}$ to be applied in both negative and positive vertical directions to study magnetization reversal processes in situ in the TEM by tilting the specimen in the presence of an applied vertical magnetic field. Although in-plane magnetic fields can, in principle, be applied using dedicated magnetizing specimen holders, such a holder was not used in the present work.

\section{Protocol}

\section{Electron microscope alignment}

1. Switch the microscope (see Table of Materials for details and Figure 1B in the representative results section) to Lorentz mode by loading a dedicated alignment file.

1. Load the specimen (e.g., a micron-sized lamella attached to a 3-mm diameter $\mathrm{Cu}$ grid; see Figure $1 \mathrm{C}$ in the representative results section for specimen preparation details; here, the investigated material is skyrmion-hosting B20-type $\mathrm{FeGe}$ ) into a TEM specimen holder (see Table of Materials for details).

2. Perform standard preparation of the microscope (e.g., filling of the cold trap) and alignment (e.g., electron beam shift, pivot points, rotation center, condenser lens astigmatism, and eucentric height of the specimen).

NOTE: Re-alignment of the microscope may be required following changes to the microscope settings (imaging mode, objective lens current, gun lens, spot size), biprism, specimen position, or temperature.

2. Correct the two-fold astigmatism of the Lorentz lens using a thin amorphous region on the specimen by monitoring the Fourier transform of such a region in real time using the image recording and processing software that is used to control the detector.

NOTE: If there is no amorphous region on the specimen, then a separate cross-grating or amorphous carbon thin film can be used for this purpose. The specimen of interest then needs to be loaded into the microscope after alignment and aberration correction have been completed. This note also applies to step 1.3.

3. If applicable, tune the image aberration corrector of the microscope using appropriate software.

NOTE: Higher-order aberration correction may not be required for electromagnetic field mapping with $\mathrm{nm}$ spatial resolution.

4. Adjust the magnification of the microscope for the desired field of view, ideally including a region of vacuum across at least $10 \%$ of the image.

5. Move the specimen away from the field of view.

1. Insert the biprism, and orient it with respect to the specimen (usually parallel to the specimen edge).

2. Apply a desired voltage to the biprism, typically at a rate of no more than $1 \mathrm{~V} / \mathrm{s}$ to avoid damage.

6. Set up an elliptical electron beam condition by tuning the condenser lens astigmatism, and center the electron beam.

NOTE: To avoid damage, do not focus the beam onto the biprism.

7. Maximize the holographic interference fringe contrast by fine-tuning the gun and condenser lens astigmatism settings.

NOTE: The fringe contrast and spacing can be monitored on the fly using acquisition control software. 
8. Wait for $15-30 \mathrm{~min}$ to allow the electron beam, microscope, biprism, and specimen to stabilize.

NOTE: The choice of magnification and biprism voltage depend on the desired field of view, spatial resolution, and signal-to-noise-ratio in the reconstructed phase.

9. Determine the magnification according to the size of the region of interest.

NOTE: A small vacuum region ( $10 \%$ of the field of view) should ideally be included in the hologram.

10. Adjust the biprism voltage according to the desired overlap width and spatial resolution.

NOTE: The electron optical spatial resolution is at best two or three times the holographic interference fringe spacing, depending on the mask size used for reconstructing the hologram (see the representative results section for details). The magnification and biprism voltage may need to be optimized iteratively. In general, the greater the magnification or the lower the biprism voltage, the better are the fringe contrast and signal-tonoise ratio in the reconstructed phase, but the poorer is the spatial resolution.

\section{Region of interest}

1. Select the area on the specimen by moving the region of interest into the field of view.

NOTE: The region of interest should normally be close to the specimen edge (or to a region of thin clean support film), as a reference wave is needed for interference with the object wave that travels through the region of interest on the specimen, and a vacuum region (ideally $\sim 10 \%$ of the field of view) should be included in the hologram.

2. If required, adjust the specimen temperature using the temperature controller of the TEM specimen holder.
Cool the cold trap of the microscope before cooling the specimen. Make sure that the column is in the best possible vacuum condition to avoid the deposition of contamination or ice on the specimen.

NOTE: Additional waiting time may be needed for stabilization of the specimen temperature.

\section{Lorentz TEM (Fresnel defocus imaging)}

1. Remanent magnetic state of the specimen

1. For Fresnel defocus imaging, switch back to round beam illumination. If required, move the biprism from the field of view.

NOTE: The condenser lens settings for round and elliptical beam illumination can typically be saved and recalled using electron microscope control software.

2. Change the defocus of the Lorentz lens (e.g., in multiples of $\pm 200 \mu \mathrm{m}$ ) to record Fresnel defocus images. Control the acquisition of defocused images by using a script in the microscope control software.

3. Set the desired exposure time, and record in-focus, underfocus, and overfocus images using the camera control software (see Figure 2 in the representative results section).

2. Field evolution of the specimen

1. Change the magnetic field applied to the specimen by tuning either the current of the conventional microscope objective lens while remaining in Lorentz mode, or if applicable, the current in the coils of a magnetizing TEM specimen holder.

2. Adjust the eucentric height of the specimen and the defocus. 
3. Check the alignment of the microscope (see section 1.1).

4. Set the exposure time, and record in-focus, underfocus, and overfocus images using the camera control software.

5. Change the applied magnetic field, specimen tilt, and/or temperature (using the temperature controller for the TEM specimen holder), as required, to follow the magnetic response of the specimen, as well as to select a suitable condition for off-axis electron holography.

\section{Off-axis electron holography}

1. Zero-field magnetic state of the specimen

1. Switch back to an elliptical beam condition, and bring the biprism to the center of the field of view.

1. Check the alignment of the specimen, biprism, and microscope.

2. Focus the specimen after waiting for $10-30 \mathrm{~min}$ for stable conditions.

2. Place the region of interest on the specimen within the field of view (see step 2.1).

3. Set the desired exposure time, and record single or multiple electron holograms of the specimen using the control software of the camera.

4. Translate the region of interest on the specimen from the field of view, and record single or multiple reference holograms using the control software of the camera.

NOTE: The exposure time and the number of holograms in steps 4.1.3 and 4.1.4 are typically chosen to be the same.
2. Field evolution of the specimen

1. Change the magnetic field applied to the specimen (see step 3.2.1)

2. Set up the elliptical beam condition, which typically depends on the objective lens setting.

3. Realign the specimen, biprism, and microscope (see step 1.6). Focus the specimen.

4. Place the region of interest within the field of view.

5. Set the desired exposure time, and record single or multiple electron holograms of the specimen using the control software of the camera.

6. Translate the region of interest on the specimen from the field of view, and record single or multiple reference holograms using the control software of the camera.

NOTE: The exposure time and the number of holograms in steps 4.2 .5 and 4.2 .6 are typically chosen to be the same.

7. Repeat the above steps for each desired value of applied field and/or applied voltage or specimen temperature, as well as before and after turning the specimen over.

1. Check whether changes in external stimuli result in instabilities of the microscope, beam, biprism, and/or specimen, and if an additional waiting time is then required to achieve stability.

2. Take particular care to design experiments that can be used to achieve a separation of the magnetic contribution to the phase from the electrostatic contribution.

NOTE: There are several ways to achieve this separation ${ }^{13}$. Here, the specimen temperature 
has been used to switch the specimen to a paramagnetic state and subsequently evaluate differences between phase images recorded at different specimen temperatures.

3. Process the recorded electron holograms digitally.

1. Reconstruct the electron holograms to calculate amplitude and phase images using commercial or home-written software.

NOTE: Normally, a Fourier-transform-based reconstruction approach is used (see the representative results section for details of hologram reconstruction).

2. Align the images in magnification, position and angle, and, if required, remove geometric distortions from them. Combine information from multiple phase images to separate the magnetic from the electrostatic contribution to the phase (see Figure 3 in the representative results section for details).

\section{Representative Results}

FThe results shown below are taken from a Lorentz microscopy and off-axis electron holography study of magnetic skyrmions in a single crystalline FeGe specimen.

\section{TEM specimen preparation. Electron-transparent} specimens of single crystalline B20-type FeGe were prepared for TEM examination using a dual-beam scanning electron microscope equipped with a Ga FIB, a micromanipulator, and gas injection systems. FIB milling was performed using 30 and $5 \mathrm{kV}$ ion beams with currents between $6.5 \mathrm{nA}$ and 47 pA. A lift-out method ${ }^{48}$ was used to fabricate a lamella, which was attached to a $\mathrm{Cu}$ grid (Figure 1C). To reduce thickness variations from curtaining, amorphous $\mathrm{C}$ was deposited on the crystal before FIB milling. Remaining ion-beam-induced damage was reduced by using low-energy ( $<1 \mathrm{keV}) \mathrm{Ar}$ ion beam sputtering ${ }^{49}$. The final specimen had approximate values of width, height, and thickness of 15,10 , and $0.1 \mu \mathrm{m}$, respectively.

Magnetic imaging-Lorentz microscopy. The magnetic state of the FeGe specimen, which is expected to follow the magnetic field $v s$ temperature phase diagram, shown in Figure 4A, was first studied using Lorentz microscopy by recording Fresnel defocus images, both at room temperature and at reduced temperature (below the Curie temperature of $\mathrm{FeGe})$.

B20-type $\mathrm{FeGe}$ is paramagnetic at room temperature. Below a transition temperature of $278 \mathrm{~K}$ (i.e., the Curie temperature), different magnetic configurations can form, depending on the applied magnetic field ${ }^{50}$. In the present study, images were recorded both at room temperature and at reduced temperature using a double-tilt, liquid-nitrogen-cooled TEM specimen holder. The specimen temperature was monitored and controlled using a temperature controller and the control software of the camera. Below the transition temperature, FeGe typically contains a helical magnetic structure in zero applied magnetic field. This magnetic texture produces mazelike lines of black and white contrast in Lorentz (Fresnel defocus) images of thin TEM specimens, as shown in Figure 4B.

Characteristic magnetic parameters of $\mathrm{FeGe}$, such as its exchange constant, Dzyaloshinskii-Moriya interaction constant, and saturation magnetization $M$, determine the equilibrium period of the helical phase $(\sim 70 \mathrm{~nm})$, as well as the critical field for magnetic saturation $(320 \mathrm{mT})$. A lattice of Bloch-type skyrmions can be formed from the helical state by applying an out-of-plane magnetic field to the specimen using slight excitation of the objection lens. In the microscope 
used in the present study, an excitation of $6 \%$ provides a field of $\sim 100 \mathrm{mT}$. The typical defocus values that are required to image a helical structure or a lattice of skyrmions are in the range of $300 \mu \mathrm{m}-1 \mathrm{~mm}$, depending on the TEM specimen thickness.

Figure 2 shows Fresnel defocus images of Bloch-type skyrmions recorded at a specimen temperature of $100 \mathrm{~K}$ in the presence of an out-of-plane magnetic field of 100 $\mathrm{mT}$, which was applied using the conventional microscope objective lens. Cooling of the specimen in the presence of an applied magnetic field results in the formation of a regular close-packed lattice of Bloch-type skyrmions ${ }^{51}$. Depending on the sign of the defocus, the contrast of each skyrmion appears as an intensity maximum or minimum, as shown in Figure 2A and Figure 2B, respectively.

Magnetic imaging-Off-axis electron holography. Off-axis electron holograms of the FeGe lamella prepared using FIB miling were recorded both at room temperature and below the critical temperature using the procedure described above.

Figure 3A shows an off-axis electron hologram recorded from B20-type FeGe at a specimen temperature of $200 \mathrm{~K}$ following cooling in the presence of a $100 \mathrm{mT}$ applied magnetic field. A voltage of $120 \mathrm{~V}$ was applied to the biprism, resulting in a holographic interference fringe spacing of $2.69 \mathrm{~nm}$ and holographic interference fringe contrast of $\sim 25 \%$.
Reconstruction of the amplitude and phase involved digitally selecting one of the side bands in the Fourier transform of the hologram (Figure 3B), masking everything outside a circular mask with a soft edge centered on the side band to zero, centering the masked side band in Fourier space, and calculating its inverse Fourier transform to provide a real-space complex wave image that contains both amplitude and phase information. The phase $\Phi=$ $\arctan (i / r)$ and amplitude $A=\sqrt{r^{2}+i^{2}}$ of the realspace complex wavefunction were evaluated from its real part $r$ and imaginary part $i$. The phase (Figure 3C) was initially evaluated modulo $2 \pi$ and therefore contained phase discontinuities, which could be unwrapped using a suitable algorithm to provide an unwrapped phase image (Figure 3D). Further details of the reconstruction procedure and corresponding open-source software can be found elsewhere $^{52,53,54}$.

A similar approach was used to record a reference hologram from vacuum alone. The reconstructed phase of the reference hologram was subtracted from that of the specimen hologram to remove phase artifacts associated with the imaging and recording system of the microscope. The specimen was then heated to room temperature, and both specimen holograms (Figure 3E) and vacuum reference holograms were recorded using the same procedure as that at reduced temperature. As FeGe is paramagnetic at room temperature, the electron optical phase shift originates purely from the electrostatic (mean inner potential) contribution to the phase. The difference between aligned phase images recorded at room temperature and reduced temperature (after correction using vacuum reference holograms) was used to provide the magnetic phase shift alone (Figure 3F). Subtraction of the phase images required sub-pixel alignment. The final 
magnetic phase image provides information about the inplane component of the magnetic induction within and around the specimen integrated in the electron beam direction (see Equation 2).

A visual representation of the projected in-plane magnetic induction can be obtained by adding contours to the magnetic phase image (e.g., by evaluating the cosine of its chosen multiple). Its derivatives can also be used to generate colors, whose hue and intensity can be used to represent the direction and magnitude of the projected in-plane magnetic induction, respectively. Figure $\mathbf{5 A}$ shows a representative magnetic phase image of Bloch-type skyrmions in $\mathrm{FeGe}$ obtained from the off-axis electron holography results shown in Figure 3. A corresponding magnetic induction map is shown in Figure 5B.

A magnetic phase image can be analyzed further to determine the projected in-plane magnetization in the specimen using either a model-independent ${ }^{55}$ or a model-based 40 algorithm. Figure 5C shows a map of in-plane magnetization determined from the magnetic phase image shown in Figure 5A using a model-based iterative reconstruction algorithm ${ }^{40}$, together with an independent measurement of the TEM specimen thickness performed using electron energy-loss spectroscopy. The magnetization is shown in units of $\mathrm{kA} /$ $\mathrm{m}$ and reveals the hexagonal shapes of the skyrmions, which result from their close-packed arrangement. The skyrmion cores, where the spins are oriented parallel to the electron beam direction, have sizes of $\sim 8 \mathrm{~nm}$. The measured magnetization peaks at a value of $\sim 135 \mathrm{kA} / \mathrm{m}$, which is in good agreement with an averaged value that considers the presence of surface twists and non-magnetic damaged specimen surface layers ${ }^{56}$. A similar approach can be used to study the evolution of the spin texture systematically as a function of applied magnetic field and temperature. 

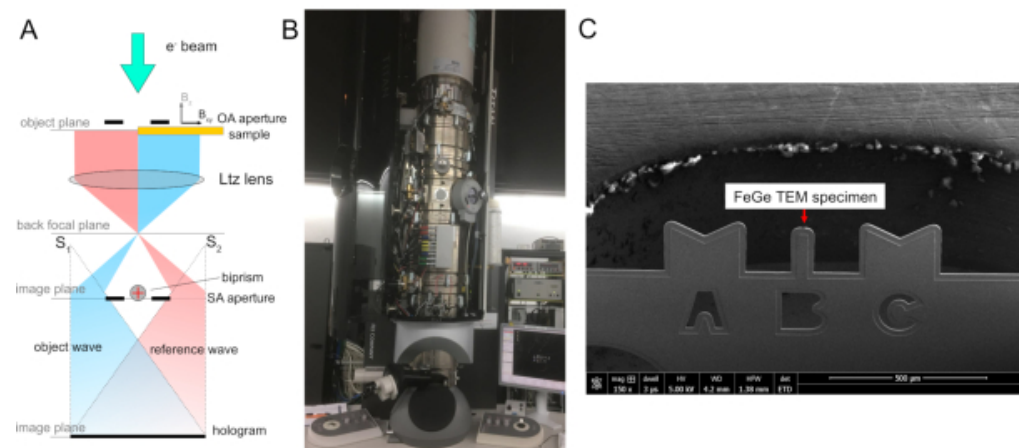

Figure 1: Basic off-axis electron holography setup and example specimen geometry. (A) Schematic ray path for off-axis electron holography. (B) Photograph of the transmission electron microscope used in this study. This microscope is equipped with a field emission gun, an image aberration corrector, a Lorentz lens, and two electron biprisms and was operated at $300 \mathrm{kV}$. (C) Secondary electron scanning electron microscopic image of a TEM specimen of B20-type FeGe, prepared using FIB miling, attached to a Cu TEM support grid (see Figure 2B for a TEM image of the specimen). Scale bar $=500 \mu \mathrm{m}$. Abbreviations: $\mathrm{OA}=$ open aperture; $\mathrm{Ltz}=$ Lorentz; $\mathrm{SA}=$ selected area; $\mathrm{e}^{-}=$electron; $\mathrm{TEM}=$ transmission electron microscope. Please click here to view a larger version of this figure.
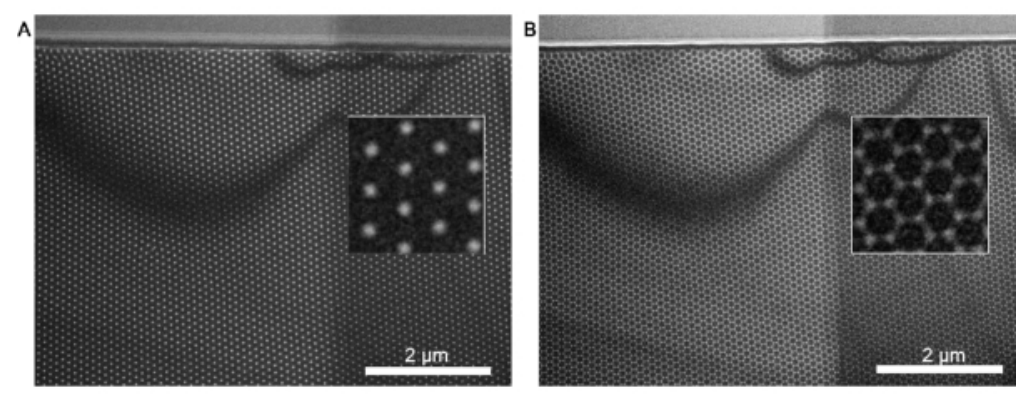

Figure 2: Fresnel defocus images of Bloch-type skyrmions in B20-type FeGe. (A) Underfocus and (B) overfocus images of Bloch-type skyrmions in an FeGe lamella prepared using FIB miling and recorded at a specimen temperature of $100 \mathrm{~K}$ in the presence of an out-of-plane magnetic field of $100 \mathrm{mT}$. The defocus values are $\pm 500 \mu \mathrm{m}$. The wide bands of wavy dark contrast are crystalline bend contours arising from diffraction contrast. The insets show magnified regions of the images. Scale bars $=2 \mu \mathrm{m}$. Please click here to view a larger version of this figure. 

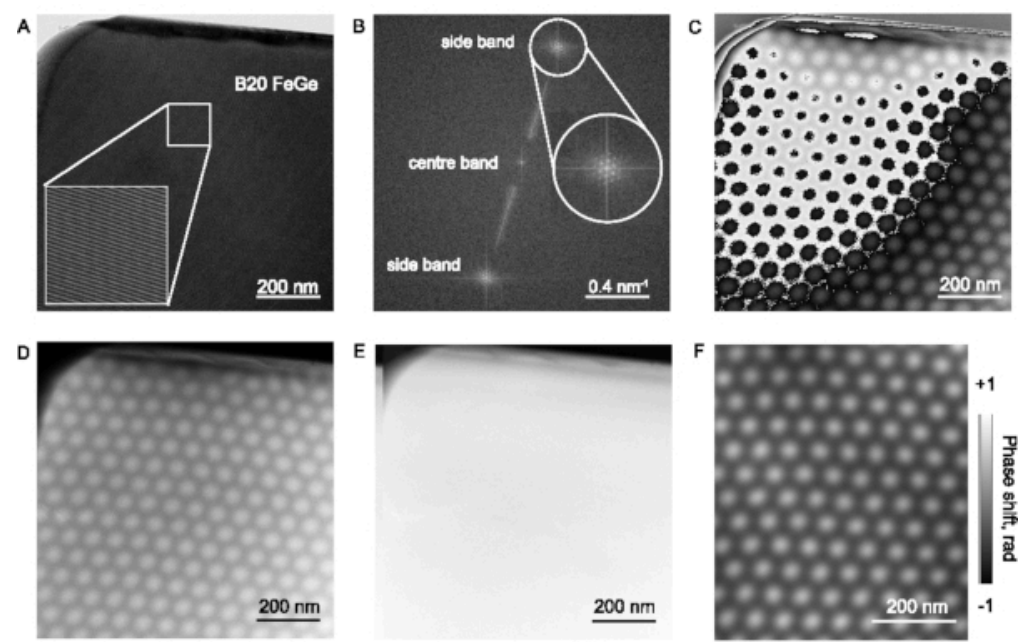

Figure 3: Reconstruction of an off-axis electron hologram. (A) Experimental off-axis electron hologram of a B20-type FeGe lamella recorded at a specimen temperature of $200 \mathrm{~K}$ in the presence of an applied out-of-plane magnetic field of $100 \mathrm{mT}$. The inset shows a magnified region of the hologram; scale bar $=200 \mathrm{~nm}$. (B) Fourier transform of the hologram containing a center band, two side bands, and streaks originating from Fresnel fringes at the edges of the biprism. An enlargement of one of the side bands reveals spots associated with the ordered arrangement of skyrmions; scale bar = $0.4 \mathrm{~nm}^{-1}$. (C) Wrapped phase image obtained by inverse Fourier transformation of one of the side bands; scale bar = $200 \mathrm{~nm}$. (D) Unwrapped phase image; scale bar $=200 \mathrm{~nm}$. (E) Unwrapped phase image of the same region recorded at room temperature in zero out-of-plane applied magnetic field; scale bar $=200 \mathrm{~nm}(\mathbf{F})$ Part of a final magnetic phase image obtained by subtracting the phase image recorded at room temperature from that recorded at $200 \mathrm{~K}$, after aligning them with sub-pixel precision; scale bar $=200 \mathrm{~nm}$. Please click here to view a larger version of this figure. 

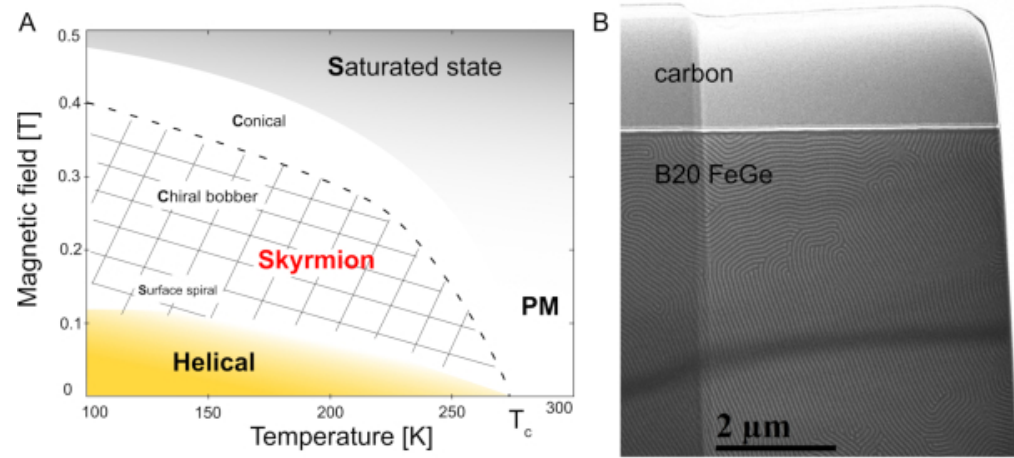

Figure 4: Magnetic field vs temperature phase diagram of FeGe. (A) Phase diagram of B20-type FeGe showing magnetic states vs temperature. (B) Fresnel defocus image of a representative helical magnetic state in an FeGe lamella prepared using FIB miling and recorded at a specimen temperature of $260 \mathrm{~K}$ in zero applied magnetic field. Scale bar $=2$ $\mu \mathrm{m}$. Abbreviations: $\mathrm{PM}=$ paramagnetic phase $; \mathrm{T}_{\mathrm{C}}=$ Curie temperature; FIBs $=$ focused ion beams. Please click here to view a larger version of this figure.
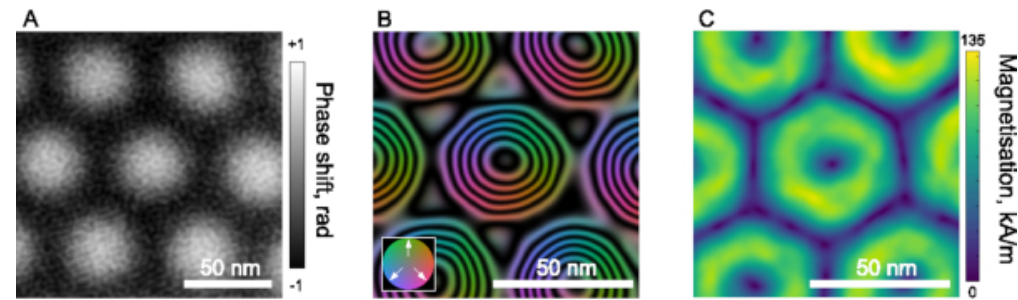

Figure 5: Quantitative analysis of magnetic phase shift. (A) Magnetic phase shift $\Phi_{m}$ of Bloch-type skyrmions in B20type FeGe recorded at a specimen temperature of $200 \mathrm{~K}$ in the presence of an applied out-of-plane magnetic field of $100 \mathrm{mT}$. (B) Magnetic induction map created by displaying the cosine of a multiple of the magnetic phase image and adding colors generated from its derivatives. The phase contour spacing is $2 \pi / 20 \sim 0.314$ radians. (C) Projected in-plane magnetization obtained from the magnetic phase image shown in $(\mathbf{A})$ using a model-based iterative reconstruction algorithm. Scale bars = $50 \mathrm{~nm}$. Please click here to view a larger version of this figure.

\section{Discussion}

Off-axis electron holography provides fully quantitative measurements of the magnetic properties of nanoscale materials with $\mathrm{nm}$ spatial resolution, either in projection or in three dimensions when combined with electron tomography.
These advantages make the technique distinct from X-ray and neutron-based techniques for the high spatial resolution characterization of magnetic nanostructures. However, care is required in the design and execution of experiments, as well as during data analysis. Some of the factors to be 
considered are mentioned here. First, magnetic materials are, in general, sensitive to ion-beam-sputtering-induced artifacts, which can result in the formation of defective, amorphous, and/or non-magnetic layers on the surface of a TEM specimen. They may also need to be stored in an inert gas atmosphere or vacuum to prevent oxidation. Furthermore, TEM specimens prepared using FIB miling are small and delicate. Therefore, mechanical tools, such as tweezers, are generally not recommended. Instead, vacuum tweezers can be used to insert specimens into TEM specimen holders.

Second, conventional cooling TEM specimen holders, such as the one used in the present study, can introduce specimen drift due to changes in the temperature of the specimen cradle, screws, and heat-transporting wires. Specimen drift typically slows down to an acceptable rate over a timeframe of 10-40 min. Third, during a cooling experiment, electronbeam-induced charging can be present, especially when studying a specimen that contains insulating materials. Charging can introduce slowly varying contributions to the electrostatic contribution to a recorded phase image, which can vary with specimen temperature, as well as with electron beam illumination and specimen position. Sometimes, coating the specimen with a thin layer of $\mathrm{C}$ can help to reduce electron-beam-induced charging.

Fourth, magnetic imaging experiments performed in the TEM require an electron-transparent specimen. The preparation of such specimens is briefly described above in the representative results section. When planning, conducting, and interpreting an experiment, the shape of the specimen must be considered, as results obtained from "thin films" may be different from those obtained from bulk samples. For example, magnetic domains are often smaller and demagnetizing fields are stronger in TEM specimens than in bulk materials. Nevertheless, magnetic properties, such as saturation magnetization and domain wall width measured from thin TEM specimens, typically match values obtained from bulk materials.

Fifth, the application of a perpendicular magnetic field to a specimen using the conventional microscope objective lens changes the image magnification and rotation, which should be corrected before digital image alignment. Slight misalignment between phase images can result in the misinterpretation of misalignment artifacts as magnetic contrast.

Looking toward the future, electron holographic tomography provides a route to the $3 \mathrm{D}$ reconstruction of magnetic fields and magnetization distributions in materials based on either backprojection-based $^{34}$ or model-based ${ }^{40}$ tomographic reconstruction algorithms. Such experiments require the acquisition and processing of large numbers of images including subtraction of the mean inner potential contribution to the phase at each specimen tilt angle. Tomographic experiments are susceptible to changes to the sample during extended experiments, changes in dynamical diffraction as a function of specimen tilt angle, artifacts resulting from the acquisition of incomplete datasets, as well as the effects of misalignment and image distortion. The automation of workflows for data acquisition and analysis promises to overcome some of these problems. Other future experimental developments may include the design of sophisticated magnetizing coils and approaches for performing time-resolved off-axis electron holography experiments of magnetic switching during the application of multiple external stimuli to specimens. 


\section{Disclosures}

The authors have nothing to disclose.

\section{Acknowledgments}

We are grateful to numerous colleagues for valuable discussions, advice, support, provision of specimens, and ongoing collaborations, as well as for funding to the European Research Council under the European Union's Horizon 2020 research and innovation programme (Grant No. 856538, project "3D MAGiC"), to the Deutsche Forschungsgemeinschaft (DFG, German Research Foundation) - Project-ID 405553726 - TRR 270, to the European Union's Horizon 2020 Research and Innovation Programme (Grant No. 823717, project "ESTEEM3"), to the European Union's Horizon 2020 Research and Innovation Programme (Grant No. 766970, project "QSORT"), and to the DARPA TEE program through grant MIPR\# HR0011831554.

\section{References}

1. Magnetic nanostructures. Eds. Aktas, B., Tagirov, L., Mikailov, F. Eds. Springer (2007).

2. Parkin, S. S. P., Hayashi, M., Thomas, L. Magnetic domain-wall racetrack memory. Science.320 (5873), 190-194 (2008).

3. Fert, A., Reyren, N., Cros, V. Magnetic skyrmions: advances in physics and potential applications. Nature Reviews Materials. 2, 17031 (2017).

4. Lavrijsen, R. et al. Magnetic ratchet for three-dimensional spintronic memory and logic. Nature. 493, 647-650 (2013).
5. Fernández-Pacheco, A. et al. Three-dimensional nanomagnetism. Nature Communications. 8, 15756 (2017).

6. Magnetic imaging and its applications to materials. De Graef, M. Ed. Academic Press (2000).

7. Modern techniques for characterizing magnetic materials. Zhu, Y. Ed. Kluwer Academic Publishers (2005).

8. Magnetic microscopy of nanostructures. Hopster, H., Oepen, H. P. Eds. Springer (2005).

9. Warwick, T. et al. A scanning transmission X-ray microscope for materials science spectromicroscopy at the advanced light source. Review of Scientific Instruments. 69, 2964-2973 (1998).

10. Faigel, G., Tegze, M. X-ray holography. Reports on Progress in Physics. 62, 355-393 (1999).

11. Izyumov, Y. A., Ozerov, R. P. Magnetic neutron diffraction. Springer, Boston, Massachusetts, USA (1995).

12. Zweck, J. Imaging of magnetic and electric fields by electron microscopy. Journal of Physics: Condensed Matter. 28, 403001 (2016).

13. Kovács, A., Dunin-Borkowski, R. E. Chapter 2-Magnetic imaging of nanostructures using off-axis electron holography. in Handbook of Magnetic Materials. Brück, E. Ed. 27, 59-153, Elsevier (2018).

14. Dunin-Borkowski, R. E., Kovács, A., Kasama, T., McCartney, M. R., Smith, D. J. Electron holography. in Springer Handbook of Microscopy. Hawkes, P. W., Spence, J. C. H. Eds. 2-2 Springer International Publishing (2019). 
15. Transmission electron microscopy: Diffraction, imaging, and spectrometry. Carter, C. B., Williams, D. B. Eds. Springer (2016).

16. Gabor, D. A new microscopic principle. Nature. 161, 777-778 (1948).

17. Cowley, J. M. Twenty forms of electron holography. Ultramicroscopy. 41 (4), 335-348 (1992).

18. Linck, M., Freitag, B., Kujawa, S., Lehmann, M., Niermann, T. State of the art in atomic resolution offaxis electron holography. Ultramicroscopy. 116, 13-23 (2012).

19. Midgley, P. A., Dunin-Borkowski, R. E. Electron tomography and holography in materials science. Nature Materials. 8, 271-280 (2009).

20. Tanigaki, T. et al. New trend in electron holography. Journal of Physics D: Applied Physics. 49 (24), 244001 (2016).

21. Lichte, $H$. et al. Electron holography: Applications to materials questions. Annual Review of Materials Research. 37, 539-588 (2007).

22. Lichte, H. et al. Electron holography: state and prospects. Microscopy and Microanalysis. 20 (S3), 244-245 (2014).

23. McCartney, M. R., Dunin-Borkowski, R. E., Smith, D. J. Quantitative measurement of nanoscale electrostatic potentials and charges using off-axis electron holography: Developments and opportunities. Ultramicroscopy. 203, 105-118 (2019).

24. Lichte, H., Lehmann, M. Electron holography-basics and applications. Reports on Progress in Physics. 71 (1), 016102 (2008).
25. Aharonov, Y., Bohm, D. Significance of electromagnetic potentials in the quantum theory. The Physical Review. 115, 485-491 (1959).

26. Zheng, F. et al. Direct imaging of a zero-field target skyrmion and its polarity switch in a chiral magnetic nanodisk. Physical Review Letters. 119, 197205 (2017).

27. Dunin-Borkowski, R. E. et al. Off-axis electron holography of magnetic nanowires and chains, rings, and planar arrays of magnetic nanoparticles. Microscopy Research and Technique. 64 (5-6), 390-402 (2004).

28. Dunin-Borkowski, R. E. et al. Magnetic microstructure of magnetotactic bacteria by electron holography. Science. 282, 1868-1870 (1998).

29. Almeida, T. P. et al. Direct visualization of the thermomagnetic behavior of pseudo-single-domain magnetite particles. Science Advances. 2 (4), e1501801 (2016).

30. Almeida, T. P. et al. Visualized effect of oxidation on magnetic recording fidelity in pseudo-single-domain magnetite particles. Nature Communications. 5, 5154 (2014).

31. Zheng, F. et al. Experimental observation of chiral magnetic bobbers in B20-type FeGe. Nature Nanotechnology. 13, 451-455 (2018).

32. Lai, G. et al. Three-dimensional reconstruction of magnetic vector fields using electron-holographic interferometry. Journal of Applied Physics. 75 (9), 4593 (1994).

33. Wolf, D., Lubk, A., Röder, F., Lichte, H. Electron holographic tomography. Current Opinion in Solid State and Materials Science. 17 (3), 126-134 (2013). 
34. Wolf, D. et al. 3D magnetic induction maps of nanoscale materials revealed by electron holographic tomography. Chemistry of Materials. 27 (19), 6771-6778 (2015).

35. Lubk, A. et al. Nanoscale three-dimensional reconstruction of electric and magnetic stray fields around nanowires. Applied Physics Letters. 105 (17), 173110 (2014).

36. Simon, P. et al. Synthesis and three-dimensional magnetic field mapping of $\mathrm{Co} 2 \mathrm{FeGa}$ heusler nanowires at $5 \mathrm{~nm}$ resolution. Nano Letters. 16 (1), 114-120 (2016).

37. Tanigaki, T. et al. Three-dimensional observation of magnetic vortex cores in stacked ferromagnetic discs. Nano Letters. 15 (2), 1309-1314 (2015).

38. Mohan, K. A., Prabhat, K. C., Phatak, C., De Graef, M., Bouman, C. A. Model-based iterative reconstruction of magnetization using vector field electron tomography. IEEE Transactions on Computational Imaging. 4 (3), 432-446 (2018).

39. Prabhat, K. C., Mohan, K. A., Phatak, C., Bouman, C., Graef, M. D. 3D reconstruction of the magnetic vector potential using model based iterative reconstruction. Ultramicroscopy. 182, 131-144 (2017).

40. Caron, J. Model-based reconstruction of magnetisation distributions in nanostructures from electron optical phase images. https://juser.fz-juelich.de/ record/851773. RWTH Aachen University. (2017).

41. Snoeck, E. et al. Off-axial aberration correction using a B-COR for Lorentz and HREM modes. Microscopy and Microanalysis. 20 (S3), 932-933 (2014).

42. Shibata, N. et al. Atomic resolution electron microscopy in a magnetic field free environment. Nature Communications. 10, 2308 (2019).
43. Phatak, C., Petford-Long, A. K., De Graef, M. Recent advances in Lorentz microscopy. Current Opinion in Solid State and Materials Science. 20 (2), 107-114 (2016).

44. Chang, S. L. Y., Dwyer, C., Barthel, J., Boothroyd, C. B., Dunin-Borkowski, R. E. Performance of a direct detection camera for off-axis electron holography. Ultramicroscopy. 161, 90-97 (2016).

45. Harada, K., Tonomura, A., Matsuda, T., Akashi, T., Togawa, Y. High-resolution observation by doublebiprism electron holography. Journal of Applied Physics. 96 (11), 6097 (2004).

46. Harada, K., Matsuda, T., Tonomura, A., Akashi, T., Togawa, Y. Triple-biprism electron interferometry. Journal of Applied Physics. 99, 113502 (2006).

47. Kasama, T., Dunin-Borkowski, R. E., Beleggia, M. Electron holography of magnetic materials. in Holography - Different Fields of Application. Ramirez, F. A. M. Ed. 53-80 InTechOpen (2011).

48. Langford, R. M., Rogers, M. In situ lift-out: Steps to improve yield and a comparison with other FIB TEM sample preparation techniques. Micron. 39 (8), 1325-1330 (2008).

49. Fischione, P. E. et al. A small spot, inert gas, ion milling process as a complementary technique to focused ion beam specimen preparation. Microscopy and Microanalysis. 23 (4), 782-793 (2017).

50. Kovács, A., Li, Z.-A., Shibata, K., Dunin-Borkowski, R. E. Lorentz microscopy and off-axis electron holography of magnetic skyrmions in FeGe. Resolution and Discovery. 1 (1), 2-8 (2016). 
51. Shibata, K. et al. Temperature and magnetic field dependence of the internal and lattice structures of skyrmions by off-axis electron holography. Physical Review Letters. 118, 087202 (2017).

52. Völkl, E., Allard, L. F., Frost, B. A software package for the processing and reconstruction of electron holograms. Journal of Microscopy. 180 (1), 39-50 (1995).

53. Niermann, T., Lehmann, M. Averaging scheme for atomic resolution off-axis electron holograms. Micron. 63, 28-34 (2014).

54. Morawiec, K., Zajkowska, W., Dłużewski, P., Shiojiri, M., Kusiński, J. PyHoLo software, a new tool for electron hologram analysis and magnetic investigation. Computer Physics Communications. 256, 107471 (2020).

55. Beleggia, M., Kasama, T., Dunin-Borkowski, R. E. The quantitative measurement of magnetic moments from phase images of nanoparticles and nanostructuresI. Fundamentals. Ultramicroscopy. 110 (5), 425-432 (2010).

56. Song, D. et al. Quantification of magnetic surface and edge states in an FeGe nanostripe by off-axis electron holography. Physical Review Letters. 120, 167204 (2018). 